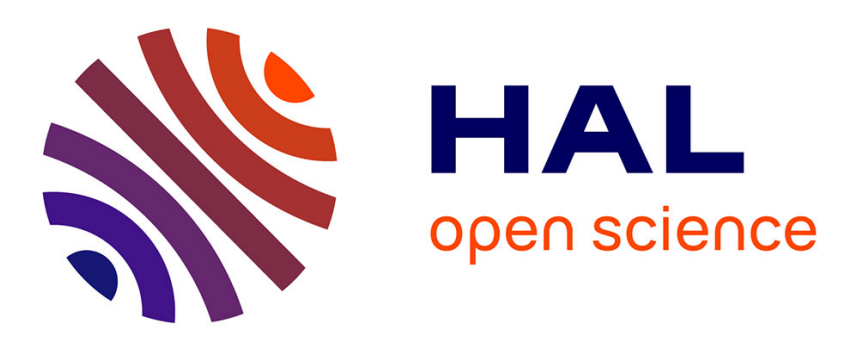

\title{
Déshydratation des laits enrichis en caséine micellaire par microfiltration; comparaison des propriétés des poudres obtenues avec celles d'une poudre de lait ultra-propre
}

\author{
P Schuck, M Piot, S Méjean, J Fauquant, G Brulé, Jl Maubois
}

\section{To cite this version:}

P Schuck, M Piot, S Méjean, J Fauquant, G Brulé, et al.. Déshydratation des laits enrichis en caséine micellaire par microfiltration; comparaison des propriétés des poudres obtenues avec celles d'une poudre de lait ultra-propre. Le Lait, 1994, 74 (1), pp.47-63. hal-00929379

\section{HAL Id: hal-00929379 https://hal.science/hal-00929379}

Submitted on 1 Jan 1994

HAL is a multi-disciplinary open access archive for the deposit and dissemination of scientific research documents, whether they are published or not. The documents may come from teaching and research institutions in France or abroad, or from public or private research centers.
L'archive ouverte pluridisciplinaire HAL, est destinée au dépôt et à la diffusion de documents scientifiques de niveau recherche, publiés ou non, émanant des établissements d'enseignement et de recherche français ou étrangers, des laboratoires publics ou privés. 


\title{
Déshydratation des laits enrichis en caséine micellaire par microfiltration; comparaison des propriétés des poudres obtenues avec celles d'une poudre de lait ultra-propre
}

\author{
P Schuck, M Piot, S Méjean, J Fauquant, G Brulé, JL Maubois
}

Laboratoire de recherches laitières, INRA, 65, rue de Saint-Brieuc, 35042 Rennes Cedex, France

(Reçu le 27 août 1993; accepté le 8 octobre 1993)

\begin{abstract}
Résumé - Le traitement du lait écrémé cru par le procédé Bactocatch, préalablement à sa concentration par évaporation et à sa déshydratation par atomisation, permet l'obtention d'une poudre de qualité très supérieure aux normes low heat : solubilité supérieure à $99,6 \%$; dispersibilité égale à $98,9 \%$; mouillabilité de $12 \mathrm{~s}$; population bactérienne égale à $3000 \mathrm{UFC} / \mathrm{g}$; temps de coagulation par la présure identique à celui du lait cru. L'enrichissement du lait en caséine micellaire par microfiltration sur membrane $0,1 \mu \mathrm{m}$ entraîne des modifications profondes des qualités physiques des poudres fabriquées à partir des rétentats. Les indices de solubilité et de dispersibilité atteignent respectivement $93,6 \%$ et $29,1 \%$ pour le rétentat de facteur de concentration volumique, $F C V=6$. Cette dégradation est probablement à relier aux mécanismes de perméation de l'eau aux interfaces : gouttelette de concentré-air, lors du séchage et granule de poudre-eau lors de la mise en solution. La modification des paramètres du séchage (abaissement des températures d'entrée et de sortie d'air) améliore la solubilité et la dispersibilité des poudres de rétentat FCV 6 qui deviennent respectivement égales à $97,2 \%$ et $43,4 \%$. Mais ces valeurs ne peuvent toutefois être considérées comme entièrement satisfaisantes et d'autres études doivent être menées. L'évolution des propriétés physiques des différentes poudres obtenues a été déterminée après 100 jours de conservation à température ambiante.
\end{abstract}

microfiltration / évaporation sous vide / séchage / lait / caséine micellaire / propriété physique / poudre / coagulation présure / viscosité / épuration bactérienne

Summary - Dehydration of an ultra-clean milk and micellar casein enriched milks. Treatment of raw skimmed milk by the Bactocatch procedure, before its concentration by vacuum evaporation and spray-drying, leads to a high quality milk powder. No heat treatment is required for obtaining a low heat powder with a bacterial count of $3000 \mathrm{CFU} / \mathrm{g}$; a solubility index as high as $99.6 \%$; a dispersibility index of $98.9 \%$ and a wettability index of $12 \mathrm{~s}$. Such a powder after water reconstitution has the same renneting time as the original raw milk and can be used as a reference powder either for industrial or scientific purposes. Enrichment of milk in micellar casein by treatment with a $0.1 \mu \mathrm{m}$ pore size diameter microfiltration membrane deeply modifies physical properties of resulting powders. According to the volumic concentration factor (VCF), solubility and dispersibility indexes decrease. Powder issued from a VCF 6 retentate has a lowered solubility and dispersibility index by 
$6 \%$ and $71 \%$ respectively. Such a degradation is probably caused by differences in water permeation during drying and reconstitution. Water transport is probably modified by the high protein content of the spraid droplet in the atomization tower and similarly when powder granule is dissolved in water. Lowering both inlet and outlet air temperatures significantly improves solubility and dispersibility indexes of micellar casein enriched milk powder. These indexes respectively increase with $97.2 \%$ and $43.3 \%$ but they are still lower than the corresponding values of reference milk powder. So, more research is needed to obtain satisfactory indexes. Effect of a 100-day storage of powders on physical properties at room temperature has been checked.

microfiltration / vacuum evaporation / spray drying / milk / micellar casein / physical properties / powder / rennet coagulation / viscosity / bacterial epuration

\section{INTRODUCTION}

Les développements récents des techniques de microfiltration sur membrane permettent d'envisager, non seulement une amélioration généralisée de la qualité bactériologique et hygiénique de l'ensemble des produits dérivés du lait (Maubois, 1991) par l'utilisation en amont de tous les processus de transformation, de la technologie Bactocatch (Vincens et Tabard, 1988), mais aussi l'obtention de nouveaux dérivés du lait par concentration sélective de la caséine micellaire (Fauquant et al, 1988 ; Pierre et al, 1992).

Les poudres de lait actuellement commercialisées, en vue de leur reconstitution sous forme de lait-boisson, dites "basse température" ou low heat doivent être issues de lait ayant subi un traitement maximal de $80^{\circ} \mathrm{C}$ pendant quelques secondes (Amariglio, 1986). Un tel traitement thermique entraîne une dénaturation des protéines solubles avec formation d'un complexe caséine $\kappa / \beta$-lactoglobuline modifiant défavorablement l'aptitude à la coagulation par la présure (Ferron-Baumy et al, 1991) des laits reconstitués. Sur un autre plan, ce traitement thermique ne provoque qu'une destruction limitée des germes thermorésistants et sporulés. II en résulte que les normes bactériologiques des laits Iow heat ADMI ou CEE sont fixées à 40000-50000 germes aérobies revivi- fiables par g de poudre (Amariglio, 1986). L'épuration du lait écrémé cru par microfiltration sur membrane $1,4 \mu \mathrm{m}$ élimine $99,6 \%$ à $99,9 \%$ de la flore contaminante en moyenne (Vincens et Tabard, 1988 ; Trouvé et al, 1991). De plus, la rétention par la membrane des bactéries thermorésistantes et sporulées est plus élevée que la rétention moyenne en raison de leur volume cellulaire supérieur (Trouvé et al, 1991). II découle de ces observations que le traitement préalable du lait, avant dessiccation par évaporation sous vide et atomisation, par le procédé Bactocatch, devrait éviter tout traitement thermique du lait et conduire à l'obtention d'une poudre de qualité supérieure aux normes low heat actuelles, notamment en ce qui concerne la qualité bactériologique. La réalisation de cette poudre en prenant en compte les résultats de Bloore et Boag (1982) sur l'influence des paramètres de déshydratation du concentré laitier a constitué l'un des objectifs de la présente étude.

Les techniques d'ultrafiltration et de microfiltration sur membrane permettent de concentrer sélectivement tout ou partie des composants protéiques du lait. Elles ont conduit à de nombreuses applications en transformation laitière et fromagère (Maubois, 1991). Elles permettent aussi d'optimiser la composition des produits transformés à leur utilisation finale, que ce soit pour répondre à des besoins de transformation fromagère dans les pays sans 
production laitière, ou pour répondre à des besoins de fonctionnalité. Or, ces débouchés potentiels en tant que matière première adaptée ou en tant qu'ingrédients alimentaires requièrent pratiquement toujours l'élimination totale de l'eau pour limiter au maximum les coûts de transport et faciliter la conservation (Maubois et Fauconneau, 1977). La sensibilité des équilibres physico-chimiques aux traitements technologiques conduit à supposer que le comportement à l'évaporation sous vide et au séchage par atomisation des rétentats de lait enrichis sélectivement en caséine micellaire est fort différent de celui observé pour le lait. II était donc nécessaire de le caractériser afin de déterminer avec précision les modifications des paramètres de concentration et de séchage à mettre en œuvre pour l'obtention de poudres présentant les propriétés optimales requises pour leur utilisation ultérieure (solubilité, dispersibilité, mouillabilité, coagulation par la présure). L'influence des conditions de concentration et de séchage de différents rétentats de microfiltration a constitué le second objectif de cette étude.

\section{MATÉRIELS ET MÉTHODES}

\section{Schéma technologique}

Le schéma technologique est représenté sur la figure 1 .

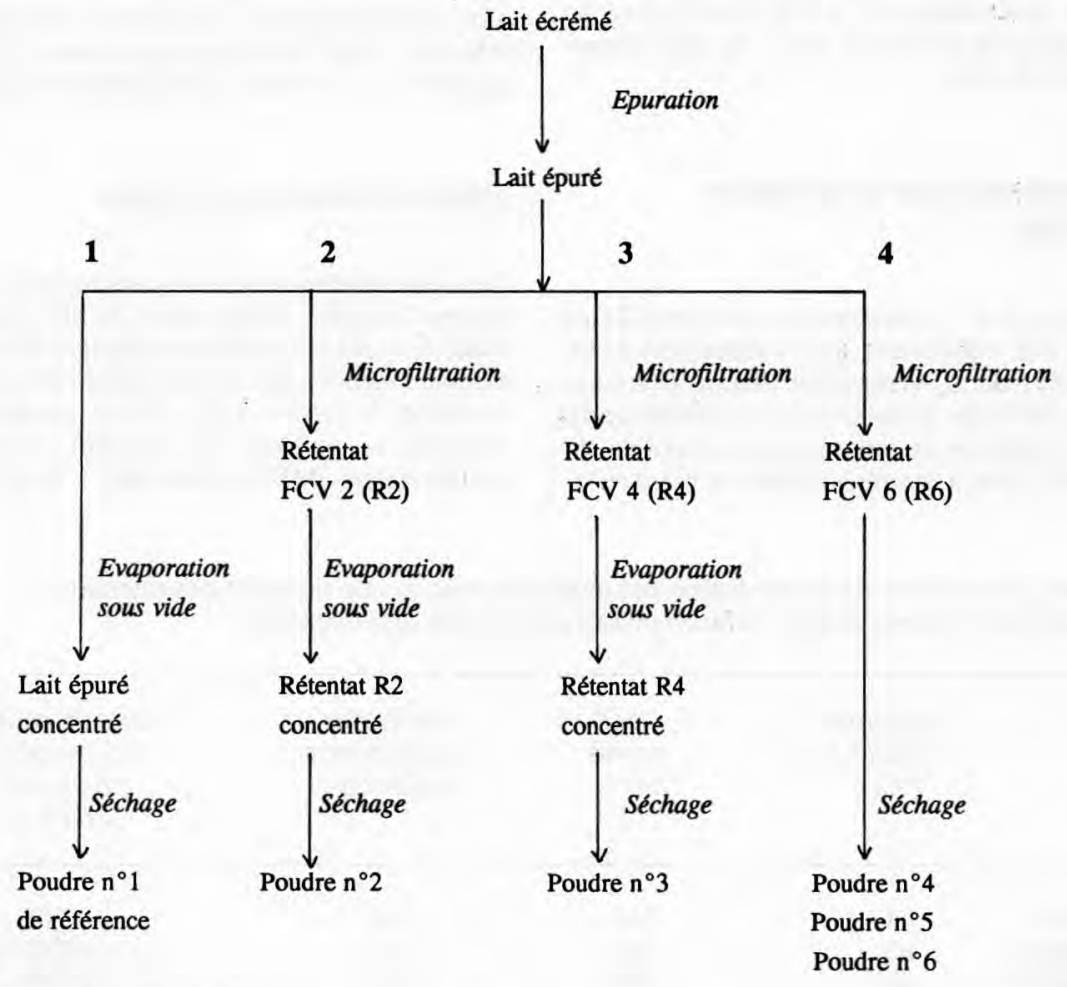

Fig 1. Schéma technologique.

Technological diagram. 


\section{Lait utilisé}

Le lait utilisé était un lait de grand mélange écrémé et cru, en provenance de la laiterie Triballat (Noyal-sur-Vilaine, France), épuré par microfiltration sur membrane minérale ayant un diamètre de pores de 1,4 $\mu \mathrm{m}$, comme l'ont décrit Trouvé et al (1991). Pour chaque essai, 1000 I ont été mis en œuvre.

\section{Microfiltration du lait épuré}

Les laits épurés $n^{\circ} 2,3$ et 4 ont été microfiltrés respectivement à des facteurs de concentration volumiques (FCV) de 2, 4 et 6 . Les membranes de microfiltration mises en œuvre étaient des membranes minérales Carbosep (TechSep Rhône-Poulenc, St-Maurice-de-Beynost, France) de type M14 (diamètre de pores de $0,14 \mu \mathrm{m}$ ). La surface totale était de $6,8 \mathrm{~m}^{2}$. La température de fonctionnement a été maintenue par l'échangeur de boucle à $50^{\circ} \mathrm{C}$. Le $\Delta \mathrm{P}$ moyen était de 0,16 MPa.

\section{Concentration par évaporation sous vide}

Le lait épuré $n^{\circ} 1$, ainsi que les rétentats $R 2$ et R4 ont été concentrés par évaporation sous vide (ESV) dans l'installation Bionov (Rennes, France) de façon à obtenir un concentré ayant un taux protéique se rapprochant de celui du rétentat R6. Cela a été réalisé dans le but d'obte- nir des concentrés ayant sensiblement la même viscosité avant leur dispersion dans l'atomiseur. L'appareil utilisé était un évaporateur sous vide double effet Laguilhare (Rueil Malmaison, France) à flot tombant, ayant une capacité évaporatoire de $180 \mathrm{l} \cdot \mathrm{h}^{-1}$. Les paramètres utilisés sont rassemblés dans le tableau I.

\section{Séchage}

Les différents concentrés ont été atomisés dans une tour de séchage MSD (Multi-Stage Dryer) ou multiples effets de la société Niro Atomizer France (Rueil Malmaison, France) ayant une capacité évaporatoire de 70 à $100 \mathrm{l} \cdot \mathrm{h}^{-1}$, dans l'installation Bionov (Rennes, France). Les principaux paramètres de fonctionnement sont indiqués dans le tableau II. Pour tous les concentrés, les fines ont été recyclées en haut de tour et les débits d'air ont été constants. La pulvérisation a été réalisée par buse sous pression de liquide avec une buse $n^{\circ} 69$ (diamètre intérieur : $0,73 \mathrm{~mm}$ ) et un pointeau $\mathrm{n}^{\circ} 421$, ce qui entraîne un angle de pulvérisation de $60^{\circ}$.

\section{Déterminations analytiques}

Les échantillons ont été caractérisés par diverses analyses telles que : le $\mathrm{pH}$, à $20^{\circ} \mathrm{C}$ à l'aide d'un pH-mètre Radiometer ayant une électrode combinée ; la matière sèche (MS) par dessiccation à l'étuve $\left(102-105^{\circ} \mathrm{C}\right)$ pendant $5 \mathrm{~h}$ (poudre) ou pendant $7 \mathrm{~h}$ (liquide); la matière azotée totale (MAT; azote total $\times 6,38$ ) par la

Tableau I. Paramètres de concentration par évaporation sous vide du lait et des rétentats. Parameters of concentration by vacuum evaporation of milk and retentates.

\begin{tabular}{lcccc}
\hline Produit & $\begin{array}{c}\text { Température } \\
\left.\text { er faisceau }^{\circ} \mathrm{C}\right)\end{array}$ & $\begin{array}{c}\text { Débit } \\
\text { entrée } \\
\left(1 \cdot h^{-1}\right)\end{array}$ & $\begin{array}{c}\text { Facteur de } \\
\text { concentration } \\
\text { volumique }\end{array}$ & $\begin{array}{c}\text { Indice de consistance } \\
\text { du concentré K } \\
\text { à } \gamma=1 \mathrm{~s}^{-1} \\
(\mathrm{mPa})\end{array}$ \\
\hline Lait épuré & 64 & 250 & 4,43 & 43,65 \\
Retentat R2 & 60 & 250 & 2,55 & 20,13 \\
Retentat R4 & 50 & 350 & 1,42 & 22,85 \\
Retentat R6 & - & - & 1,00 & 23,50 \\
\hline
\end{tabular}


Tableau II. Conditions de séchage des concentrés.

Drying parameters of the concentrates.

\begin{tabular}{|c|c|c|c|c|c|c|c|c|}
\hline Produit & $\begin{array}{l}\text { Température } \\
\text { air entrée } \\
\left({ }^{\circ} \mathrm{C}\right)\end{array}$ & $\begin{array}{l}\text { Température } \\
\text { air sortie } \\
\left({ }^{\circ} \mathrm{C}\right)\end{array}$ & $\begin{array}{l}\text { Température } \\
\text { air lit statique } \\
\left({ }^{\circ} \mathrm{C}\right)\end{array}$ & $\begin{array}{l}\text { Pression de } \\
\text { pulvérisation } \\
\text { (MPa) }\end{array}$ & $\begin{array}{l}\text { Température } \\
\text { air VF1 a } \\
\left({ }^{\circ} \mathrm{C}\right)\end{array}$ & $\begin{array}{l}\text { Température } \\
\text { air VF2 }{ }^{b} \\
\left({ }^{\circ} \mathrm{C}\right)\end{array}$ & $\begin{array}{l}\text { Hauteur lit } \\
\text { statique } \\
(\%)\end{array}$ & $\begin{array}{l}\text { Poudre } \\
n^{\circ}\end{array}$ \\
\hline Lait ESV & 200 & 77 & 70 & 16 & 50 & 35 & 15 à 20 & 1 \\
\hline R2 ESV & 220 & 77 & 70 & 16 & 50 & 35 & 15 à 20 & 2 \\
\hline R4 ESV & 233 & 77 & 70 & 16 & 50 & 35 & 15 à 20 & 3 \\
\hline $\mathrm{R} 6$ & 240 & 77 & 70 & 16 & 50 & 35 & 15 à 20 & 4 \\
\hline $\mathrm{R} 6$ & 210 & 77 & 70 & 11 & 50 & 35 & 15 à 20 & 5 \\
\hline R6 & 140 & 45 & 70 & 16 & 50 & 35 & 15 à 20 & 6 \\
\hline
\end{tabular}

a Température de l'air au niveau de la $1^{\text {re }}$ partie du vibro-fluidiseur. ${ }^{\mathrm{b}}$ Température de l'air au niveau de la $2^{\mathrm{e}}$ partie du vibro-fluidiseur.

a Air temperature in the first part of the vibrofluidizer. ${ }^{b}$ Air temperature in the second part of the vibrofluidizer 
méthode de Kjeldahl et à l'aide du Técator (Humeau, Nantes, France) ; l'azote non caséinique (NCN) par détermination de la teneur en azote total du surnageant obtenu après précipitation isoélectrique des caséines, selon la méthode de Aschaffenburg et Drewry (1959) (si le rapport NCN/MAT > $18 \%$, la poudre est considérée comme low heat) ; l'azote non protéinique (NPN) par dosage de l'azote total du surnageant obtenu après précipitation de la totalité des protéines en présence d'acide trichloracétique à $12 \%$ (Rowland, 1938) ; l'indice des protéines solubles (WPNI), par différence entre le NCN et le NPN (Alais, 1984) ; le lactose (LACT) par la méthode décrite par Acton (1977) ; les cendres par incinération de la matière sèche dans un four à $550^{\circ} \mathrm{C}$ selon la méthode de I'AOAC (Standards, 1945); le calcium total $\left(\mathrm{Ca}_{1}\right)$ et le calcium soluble $\left(\mathrm{Ca}_{\text {.s }}\right)$ par spectrophotométrie d'absorption atomique sur appareil Varian AA 300 (Les Ulis, France) selon le protocole décrit par Murthy et Rhea (1967), modifié par Brulé et al (1974).

Les analyses microbiologiques ont été réalisées selon la norme AFNOR NF V 04-015 (1984).

Les mesures rhéologiques des concentrés ont été effectuées à $40^{\circ} \mathrm{C}$ à l'aide d'un viscosimètre rotatif à cylindres coaxiaux Rhéomat 30 . À partir de la loi de Newton : $\sigma=K \cdot(\gamma)^{n}$ où $\sigma$ est la contrainte $; \mathrm{K}$, l'indice de consistance $; \gamma$, la vitesse de déformation et $n$, lindice de comportement et sachant que $\eta=\sigma / \gamma$, nous avons déterminé les indices $\mathrm{K}$ et $\mathrm{n}$ d'après la représentation graphique $\log \eta=\log K+(n-1) \cdot \log \gamma$.

La caractérisation physique des poudres a été réalisée par les mesures: de masse volumique apparente selon la méthode $n^{\circ}$ A2a de Niro Atomizer (1978); de solubilité selon la méthode normalisée de la FIL (1988), (le résultat est donné en $\%(\mathrm{v} / \mathrm{v})$ de matière soluble) ; de dispersibilité selon la méthode $n^{\circ} A 6 c$ de Niro Atomizer (1978); de mouillabilité selon la méthode $n^{\circ}$ A5a de Niro Atomizer (1978). La détermination de l'activité de l'eau $\left(\mathrm{a}_{\mathrm{w}}\right)$ et le tracé de l'isotherme d'adsorption à $25^{\circ} \mathrm{C}$ ont été réalisés à l'aide d'un aw-mètre Novasina RTD-33 TH-2 thermostaté (Pfäffikon, Suisse) et les profils granulométriques des poudres à l'aide d'un tamiseur à courant d'air Alpine A 200 LS (Évry, France) (20 g, $3 \mathrm{~min}$ ). La plupart de ces mesures permettent de caractériser l'instantanèité d'une poudre (solubilité $>99 \%$, dispersibilité > $99 \%$, mouillabilité $<20$ s).
Les poudres ont été stockées dans des flacons identiques (à base de polystyrène, épaisseur $0,4 \mathrm{~mm}$ ) et dans les mêmes conditions ambiantes d'humidité (55 à $60 \%$ d'humidité relative, $\mathrm{HR}$ ) et de température $\left(20 \mathrm{a} 25^{\circ} \mathrm{C}\right)$. Au bout de $100 \mathrm{j}$, nous avons analysé à nouveau ces poudres (solubilité, dispersibilité, mouillabilité, $\mathrm{a}_{\mathrm{w}}$ et humidité).

L'aptitude à la coagulation présure des laits, laits concentrés, rétentats, rétentats concentrés et concentrés reconstitués à partir des poudres a été appréciée à l'aide du Formagraph (Foss Electric, Paris, France) par la mesure du temps de prise $(\mathrm{R})$, du $\mathrm{K}_{20}$ (temps pour obtenir un écartement de $20 \mathrm{~mm}$ permettant d'évaluer la vitesse de raffermissement) et de la fermeté du coagulum ( $\mathrm{aR}$; écartement équivalent à une fois le temps de prise) (Zannoni et Annibaldi, 1981 ; Mc Mahon et Brown, 1982). Pour cette mesure, les différents échantillons ont été standardisés en caséine à $25 \mathrm{~g} \cdot \mathrm{H}^{-1}$ dans de l'eau distillée, agités pendant $15 \mathrm{~min}$, maintenus $1 \mathrm{~h}$ au bain-marie puis ajustés à $\mathrm{pH} 6,6$ et à $30^{\circ} \mathrm{C}$ sous agitation à l'aide d'une solution d'acide chlorhydrique $\mathrm{N}$. Après 16 à $18 \mathrm{~h}$ à $30^{\circ} \mathrm{C}$, le $\mathrm{pH}$ a été contrôlé et ajusté à nouveau à 6,6 si nécessaire. Le Formagraph a été stabilisé à $30^{\circ} \mathrm{C}$ pendant 30 min minimum. Chacune des 10 cuves a été remplie de $10 \mathrm{ml}$ d'échantillon. Après $15 \mathrm{~min}$ d'équilibrage, $100 \mu \mathrm{l}$ de solution de présure à $0,5 \%$ ont été mélangés à chaque échantillon.

\section{RÉSULTATS ET DISCUSSION}

\section{Poudre de lait de référence (Poudre $n^{\circ}$ 1)}

Les caractéristiques physico-chimiques du lait mis en cuvre et des différents produits obtenus lors de cette fabrication sont présentées dans le tableau III. L'épuration bactérienne du lait cru par microfiltration tangentielle conduit à un abaissement de $0,4 \mathrm{~g} / \mathrm{kg}$ de la teneur en matière sèche, en raison de la rétention par la membrane de la matière grasse globulaire résiduelle du lait écrémé et d'une petite proportion des grosses micelles de caséine (Maubois, 1991). La concentration de l'ensemble des 
Tableau III. Composition chimique des différents produits obtenus pour la fabrication de la poudre de lait écrémé de référence.

Chemical composition of the different products obtained for the manufacture of the reference skimmilk powder.

\begin{tabular}{|c|c|c|c|c|c|c|c|c|c|c|c|}
\hline $\begin{array}{l}N^{\circ} \\
\text { étape }\end{array}$ & Produit & $p H$ & $\begin{array}{c}M S \\
(g / k g)\end{array}$ & $\begin{array}{l}\text { MAT } \\
(g / k g)\end{array}$ & $\begin{array}{l}N C N \\
(g / k g)\end{array}$ & $\begin{array}{l}N P N \\
(g / k g)\end{array}$ & $\begin{array}{l}L A C T \\
(g / k g)\end{array}$ & $\begin{array}{l}\text { Cendres } \\
(\mathrm{g} / \mathrm{kg})\end{array}$ & $\begin{array}{l}s \quad C a . t \\
(m g / k g)\end{array}$ & $\begin{array}{c}\mathrm{Ca}_{\mathrm{s}_{\mathrm{s}}} \mathrm{C} \\
(\mathrm{mg} / \mathrm{kg})\end{array}$ & $\begin{array}{l}\text { Ca.s } / \mathrm{Ca}_{\text {. }} \\
\text { ) (\%) }\end{array}$ \\
\hline & Lait écrémé & 6,63 & 89,32 & 33,10 & 7,54 & 1,94 & 47,78 & 7,57 & 1219 & 359 & 29,45 \\
\hline & $\begin{array}{l}\text { Lait ecrémé } \\
\text { épuré }\end{array}$ & 6,63 & 88,91 & 32,85 & 7,55 & 1,94 & 47,19 & 7,57 & 1213 & 344 & 28,36 \\
\hline 1 & Lait ESV & 6,14 & 395,37 & 145,73 & 33,00 & 8,75 & 215,20 & 33,40 & 5490 & 1218 & 22,18 \\
\hline 1 & $\begin{array}{l}\text { Poudre } \\
N^{\circ} 1\end{array}$ & $6,71^{a}$ & 942,80 & 348,00 & 78,75 & 20,10 & 541,60 & 79,50 & $5389^{b}$ & $1480^{b}$ & 27,46 \\
\hline
\end{tabular}

\footnotetext{
a Mesure effectuée sur le lait reconstitué (à $10 \%$ ), à partir de la poudre $n^{\circ} 1 ;{ }^{b}$ mesure effectuée sur le lait reconstitué (équivalent en MS au lait ESV), à partir de la poudre $n^{\circ} 1$.

${ }^{a}$ Measured on milk reconstituted (10\%) from powder $n^{\circ} 1 .{ }^{b}$ Measured on milk reconstituted (equivalent in total solids to vacuum evaporated milk) from powder $n^{\circ} 1$.
}

éléments du lait par évaporation sous vide abaisse le $\mathrm{pH}$ de 0,5 unité et décroît le rapport $\mathrm{Ca} . s / \mathrm{Ca}_{\mathrm{t}}$ de $6,2 \%$. Ces variations de paramètres physico-chimiques ne sont pas retrouvées dans le lait reconstitué à partir de la poudre.

Les caractéristiques physiques de cette poudre sont présentées dans le tableau IV. Elles se situent à l'intérieur des fourchettes de variation habituellement constatées pour les poudres issues d'installations de grosse capacité (Borios et al, 1984 ; Tuohy, 1989 ; llari et Loisel, 1991).

La flore aérobie mésophile revivifiable de la poudre $n^{\circ} 1$ était de 3000 UFC/g. La population coliforme était inférieure à 5 UFC/g et aucun germe staphylocoque toxinogène n'était détecté par $\mathrm{g}$ de poudre.

L'aptitude à la coagulation par la présure de la poudre reconstituée est caracté- risée par les résultats rassemblés dans le tableau V. Aucune différence significative n'était constatée sur le temps de prise des différents laits, ce qui est en parfait accord avec la non-dénaturation des protéines solubles observée (rapports NCN/MAT identiques). En revanche, la concentration par évaporation et l'atomisation accroissent le $\mathrm{K}_{20}$ de 34 et $36 \%$ respectivement par rapport au lait épuré et décroissent la fermeté des gels de $9,5 \%$ et de $14 \%$ respectivement. Ces 2 observations sont probablement à relier aux déplacements partiellement réversibles des sels phosphocalciques de la phase soluble vers la phase colloïdale entraînés par l'évaporation sous vide et le séchage par atomisation (Le Graet et Brulé, 1982).

La poudre de lait $n^{\circ} 1$, obtenue lors de cette étude, est sans conteste une poudre de qualité low heat. Son WPNI est en effet 
Tableau IV: Caractéristiques physiques et humidité des poudres à $\mathrm{J}$ et $\mathrm{J}+100$. Physical characteristics and moisture of the powders.

\begin{tabular}{|c|c|c|c|c|c|c|c|c|c|c|c|}
\hline \multirow[t]{2}{*}{ Poudre } & $\begin{array}{l}\text { Masse } \\
\text { volumique } \\
\text { apparente } \\
\left(\mathrm{g} / \mathrm{cm}^{3}\right)\end{array}$ & \multicolumn{2}{|c|}{$\begin{array}{l}\text { Solubilité } \\
(\%, v / v)\end{array}$} & \multicolumn{2}{|c|}{$\begin{array}{c}\text { Dispersibilité } \\
(\%, p / p)\end{array}$} & \multicolumn{2}{|c|}{$\begin{array}{l}\text { Mouillabilité } \\
\text { (s) }\end{array}$} & \multicolumn{2}{|c|}{$\begin{array}{l}\text { Activité } \\
\text { de l'eau }\end{array}$} & \multicolumn{2}{|c|}{$\begin{array}{l}\text { Humidité } \\
\text { (\%) }\end{array}$} \\
\hline & $J$ & $J$ & $J+100$ & $J$ & $J+100$ & $J$ & $J+100$ & $J$ & $J+100$ & J & $J+100$ \\
\hline 1 & 0,404 & $>99,60$ & $>99,60$ & 98,94 & 98,60 & 11 & 12 & 0,363 & 0,403 & 5,72 & 6,77 \\
\hline 2 & 0,432 & $>99,60$ & 99,40 & 98,47 & 96,98 & 15 & 14 & 0,349 & 0,401 & 5,00 & 6,29 \\
\hline 3 & 0,431 & 99,40 & 98,25 & 80,55 & 69,21 & 13 & 15 & 0,347 & 0,421 & 5,09 & 6,55 \\
\hline 4 & 0,430 & 93,60 & 84,50 & 29,10 & 27,22 & 14 & 11 & 0,350 & 0,426 & 5,53 & 7,12 \\
\hline 5 & 0,413 & 94,80 & 85,50 & 40,41 & 37,42 & 15 & 14 & 0,360 & 0,401 & 4,74 & 6,67 \\
\hline 6 & 0,393 & 97,20 & 92,50 & 43,37 & 41,04 & 12 & 11 & 0,450 & 0,492 & 8,28 & 8,82 \\
\hline
\end{tabular}

égal à 9,2 mg d'azote/g de poudre (Alais, 1984). Son temps de coagulation par la présure est identique à celui constaté pour le lait cru dont elle est issue. Sa qualité bactériologique est élevée ( 300 UFC/ml de lait reconstitué). Ses caractéristiques physiques (solubilité, dispersibilité et mouilla- bilité) sont similaires à celles d'une poudre «instantanée» (De Vilder, 1986). L'ensemble de ces propriétés résulte de l'utilisation successive d'une épuration bactérienne par microfiltration sur membrane éliminant la majeure partie de la flore contaminante du lait cru (Trouvé et al,

Tableau V. Aptitude à la coagulation des différents produits obtenus pour la fabrication de la poudre de lait écrémé de référence.

Coagulation properties of the different products obtained for the manufacture of the reference skimmilk powder.

\begin{tabular}{llll}
\hline Produit ${ }^{1}$ & $\begin{array}{l}\text { Temps de prise }(R) \\
(\mathrm{min})\end{array}$ & $\begin{array}{l}K_{20} \\
(\mathrm{~min})\end{array}$ & $\begin{array}{l}\text { Fermeté }(\mathrm{aR}) \\
(\mathrm{min})\end{array}$ \\
\hline Lait écrémé & 18,87 & 10,31 & 28,30 \\
Lait écrémé épuré & 18,75 & 10,25 & 28,10 \\
Lait ESV & 18,77 & 13,80 & 25,60 \\
Poudre No1 & 18,80 & 14,07 & 24,40 \\
\hline
\end{tabular}

\footnotetext{
${ }^{1}$ Tous ces produits ont été reconstitués et standardisés en caséines à $25 \mathrm{~g} \cdot \mathrm{l}^{-1}$ dans de l'eau distillée.

${ }^{1}$ Products reconstituted and standardized in casein to $25 \mathrm{~g} \cdot{ }^{-1}$ in distilled water.
} 
1991) et de la concentration-séchage du lait ainsi épuré à l'aide d'une installation pilote «multiple effet" à traitement thermique pratiquement non dénaturant. Une telle poudre pourrait avantageusement servir de substrat de référence pour des mesures de temps de coagulation ou de vitesse d'acidification.

\section{Influence de l'enrichissement en caséine micellaire}

Le tableau VI rassemble les résultats d'analyse biochimique des rétentats de microfiltration sur membrane $0,14 \mu \mathrm{m}$ à différents FCV, avant et après concentration

Tableau VI. Composition chimique des différents produits obtenus pour la fabrication des poudres de rétentat.

Chemical composition of the different products obtained for the manufacture of retentate powders.

\begin{tabular}{|c|c|c|c|c|c|c|c|c|c|c|c|}
\hline $\begin{array}{l}N^{\circ} \\
\text { étape }\end{array}$ & Produit & $p H$ & $\begin{array}{c}M S \\
(g / k g)\end{array}$ & $\begin{array}{c}\text { MAT } \\
(g / k g)\end{array}$ & $\begin{array}{l}N C N \\
(g / k g)\end{array}$ & $\begin{array}{l}N P N \\
(g / k g)\end{array}$ & $\begin{array}{l}L A C T \\
(g / k g)\end{array}$ & $\begin{array}{l}\text { Cendre } \\
(\mathrm{g} / \mathrm{kg})\end{array}$ & $\begin{array}{c}C a_{t_{t}} \\
(\mathrm{mg} / \mathrm{kg})\end{array}$ & $\begin{array}{c}\mathrm{Ca}_{\mathrm{s}} \\
(\mathrm{mg} / \mathrm{kg})\end{array}$ & $\begin{array}{l}\mathrm{Ca} \cdot{ }_{t} / \mathrm{Ca}{ }_{s} \\
\text { (\%) }\end{array}$ \\
\hline 2 & Rétentat R2 & 6,60 & 116,65 & 61,00 & 10,11 & 1,93 & 43,80 & 10,10 & 2098 & 336 & 16,01 \\
\hline 3 & Rétentat R4 & 6,60 & 170,10 & 111,25 & 13,24 & 2,04 & 42,18 & 14,87 & 3804 & 340 & 8,94 \\
\hline 4 & Rétentat R6 & 6,61 & 212,90 & 154,70 & 15,45 & 2,13 & 38,20 & 18,82 & 5282 & 322 & 6,10 \\
\hline 2 & $\begin{array}{l}\text { Rétentat R2 } \\
\text { ESV }\end{array}$ & 6,40 & 297,85 & 157,40 & 25,70 & 5,02 & 116,10 & 25,55 & 5403 & 657 & 12,16 \\
\hline 3 & $\begin{array}{l}\text { Rétentat R4 } \\
\text { ESV }\end{array}$ & 6,53 & 241,00 & 159,00 & 18,43 & 2,91 & 60,60 & 21,40 & 5351 & 420 & 7,85 \\
\hline 2 & Poudre $\mathrm{N}^{\circ} 2$ & $6,70^{a}$ & 950,00 & 495,30 & 82,81 & 15,66 & 380,22 & 80,45 & $5219^{b}$ & $837^{b}$ & 16,04 \\
\hline 3 & Poudre $\mathrm{N}^{\circ} 3$ & $6,73^{a}$ & 949,10 & 623,40 & 73,93 & 10,96 & 242,22 & 83,60 & $5310^{\circ}$ & $534^{c}$ & 10,05 \\
\hline 4 & Poudre $\mathrm{N}^{\circ} 4$ & $6,75^{a}$ & 944,70 & 678,80 & 68,60 & 8,84 & 186,34 & 83,70 & $5246^{d}$ & $303^{d}$ & 5,77 \\
\hline 4 & Poudre $N^{\circ} 5$ & $6,76^{a}$ & 952,60 & 681,00 & 68,63 & 9,03 & 181,30 & 84,00 & $5203^{d}$ & $315^{d}$ & 6,05 \\
\hline 4 & Poudre $N^{\circ} 6$ & $6,71^{a}$ & 917,20 & 661,70 & 67,15 & 8,59 & 175,00 & 81,20 & $5262^{d}$ & $313^{d}$ & 5,95 \\
\hline
\end{tabular}

\footnotetext{
${ }^{a}$ Mesure effectuée sur le lait reconstitué (à $10 \%$ ) à partir de la poudre correspondante. ${ }^{b}$ Mesure effectuée sur le rétentat reconstitué (équivalent en MS au rétentat R2 ESV) à partir de la poudre $n^{\circ} 2 .{ }^{\circ}$ Mesure effectuée sur le rétentat reconstitué (équivalent en MS au rétentat R4 ESV) à partir de la poudre $n^{\circ} 3$. d: Mesure effectuée sur le rétentat reconstitué (équivalent en MS au rétentat R6) à partir de la poudre correspondante.

${ }^{a}$ Measured on milk reconstituted (10\%) from the corresponding powder, ${ }^{b}$ Measured on retentate reconstituted (equivalent in total solids to vacuum evaporated $R 2$ retentate) from powder $n^{\circ} 2 .{ }^{c}$ Measured on retentate reconstituted (equivalent in total solids to vacuum evaporated $R 4$ retentate) from powder $n^{\circ} 3$. $^{b}$ : Measured on retentate reconstituted (equivalent in total solids to $R 6$ retentate) from the corresponding powder.
} 
par évaporation (R2 et R4), et des poudres qui en sont issues. Comme attendu et décrit par Fauquant et al (1988), la concentration sélective de la caséine micellaire par microfiltration sur membrane accroit les rapports MAT/MS et (MATNCN)/MAT des rétentats en fonction du FCV. Ce dernier rapport est égal à $83,4 \%$ pour le R2 ; il atteint plus de $90 \%$ pour le R6, alors qu'il est égal à $77,2 \%$ dans le lait initial. Le passage de la phase aqueuse du lait au travers de la membrane de microfiltration entraîne comme pour les rétentats d'ultrafiltration une décroissance des teneurs en lactose et des rapports NPN/ MAT et $\mathrm{Ca}$.s $/ \mathrm{Ca}$.t. $\mathrm{Ce}$ dernier rapport, proche de $29 \%$ dans le lait initial décroît à $15,6 \%$ dans le R2, à $9 \%$ dans le R4 et à $6 \%$ dans le $\mathrm{R} 6$. Le $\mathrm{pH}$ des rétentats n'est pas influencé par l'élévation du FCV. En revanche, l'évaporation sous vide entraîne une baisse de 0,2 unité $\mathrm{pH}$ pour le $\mathrm{R} 2$ et de 0,07 unité pour le R4 pour une même teneur finale en MAT (157 g/kg). Ces évolutions du $\mathrm{pH}$ sont la résultante d'un accroissement de la force ionique liée à l'évaporation et de l'élévation du pouvoir tampon des rétentats de microfiltration qui doit être proche de celle observée par Brulé et al (1974) pour les rétentats d'ultrafiltration.

Le taux de MAT de tous les produits avant atomisation était voisin de celui du rétentat R6 (entre 145 et $159 \mathrm{~g} / \mathrm{kg}$ ). L'objectif poursuivi était l'obtention d'une viscosité similaire à l'entrée en tour d'atomisation. Les mesures de viscosité réalisées sont présentées sur la figure 2. Le lait évaporé a la viscosité la plus élevée : 18,7 $\mathrm{mPa}$.s pour une vitesse de déformation $\gamma$ égale à $82 \mathrm{~s}^{-1}$. Son indice de consistance $\mathrm{K}$ est égal à $43,65 \mathrm{mPa}$.s pour $\gamma=1 \mathrm{~s}^{-1}$. Son comportement est rhéofluidifiant. Ces résultats sont en accord avec ceux de Culioli et al (1974). Les 3 autres concentrés ont une viscosité nettement plus faible (entre 5,8 et $8,0 \mathrm{mPa}$.s pour $\gamma=82 \mathrm{~s}^{-1}$ ).
Leurs indices de consistance $\mathrm{K}$ sont compris entre 20 et $24 \mathrm{mPa} . \mathrm{s}$ pour $\gamma=1 \mathrm{~s}^{-1}$. Leurs comportements sont également rhéofluidifiants $(n<1)$. La différence de viscosité entre ces 3 concentrés et le lait ESV n'a vraisemblablement pas modifié la taille de la gouttelette, car d'après Morand (1975) et Masters (1991), cette taille ne varie qu'avec la racine sixième de la viscosité. En première analyse, le comportement rhéologique des laits enrichis en caséine micellaire apparaît très similaire à celui des rétentats d'ultrafiltration (Culioli et al, 1974). La caséine micellaire a donc un rôle dominant dans l'effet de la teneur en protéines sur la viscosité. La pulvérisation par buse de ces concentrés entraîne une baisse de viscosité de 15,5 mPa.s pour le lait ESV, de $6,0 \mathrm{mPa}$.s pour R2 ESV, de $9,15 \mathrm{mPa}$.s pour R4 ESV et seulement de $0,6 \mathrm{mPa}$.s pour R6. Cette résistance originale au cisaillement des laits enrichis en caséine micellaire mériterait d'être approfondie.

L'atomisation des rétentats R2 ESV, R4 ESV et R6 a été effectuée dans les mêmes conditions que celles du lait ESV (tableau II). Comme constaté pour la poudre $n^{\circ} 1$, les conditions de séchage mises en cuvre n'entraînent pas de dénaturation thermique des protéines solubles des concentrés (les rapports NCN/MS et NCN/MAT ne présentent pas d'évolution significative pour chaque couple concentré-poudre). Le rapport $\mathrm{Ca} .{ }_{s} / \mathrm{Ca}_{1}$ décroît de $15,6 \%$ à $12,2 \%$ et de $9,0 \%$ à $7,6 \%$ lors de l'évaporation respective des R2 et R4 mais retrouve sa valeur initiale et même légèrement supérieure lors de la reconstitution des laits à partir des poudres. Ces observations sont conformes à celles réalisées par Le Graet et Brulé (1982) sur le séchage des rétentats d'ultrafiltration qui avaient émis l'hypothèse d'une légère solubilisation du calcium micellaire lors du séchage dépendant de la teneur initiale en protéines du rétentat. Cette resolubilisation 
Fig 2. Viscosité des concentrés avant et après pulvérisation.

Viscosity of concentrates before and after spray-drying.

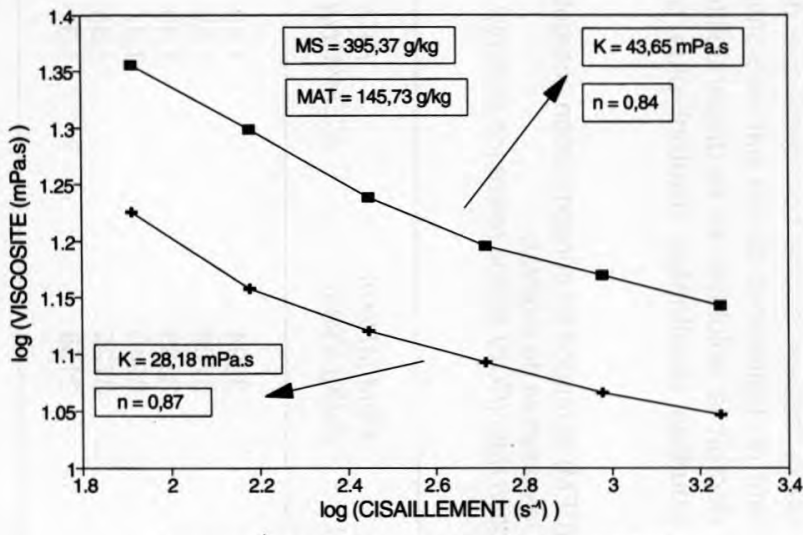

- - LAIT ESV (avant) - + LAIT ESV (après)

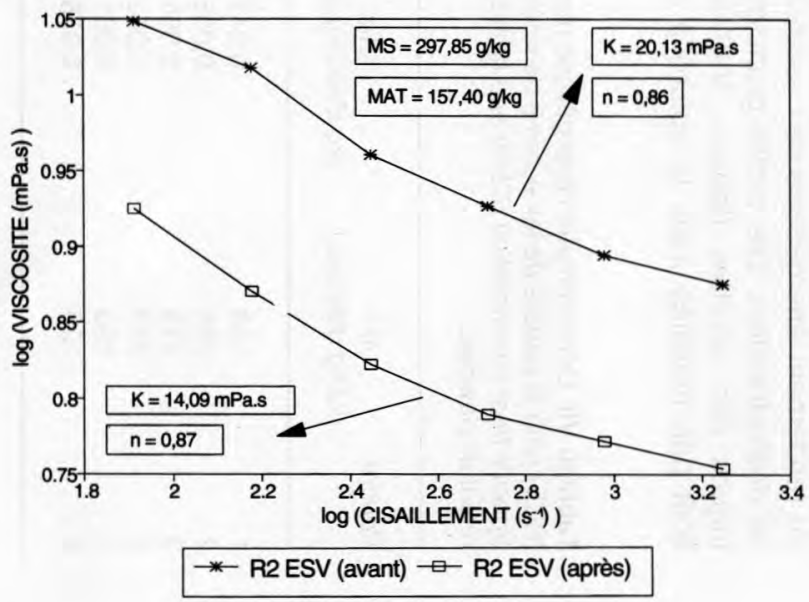

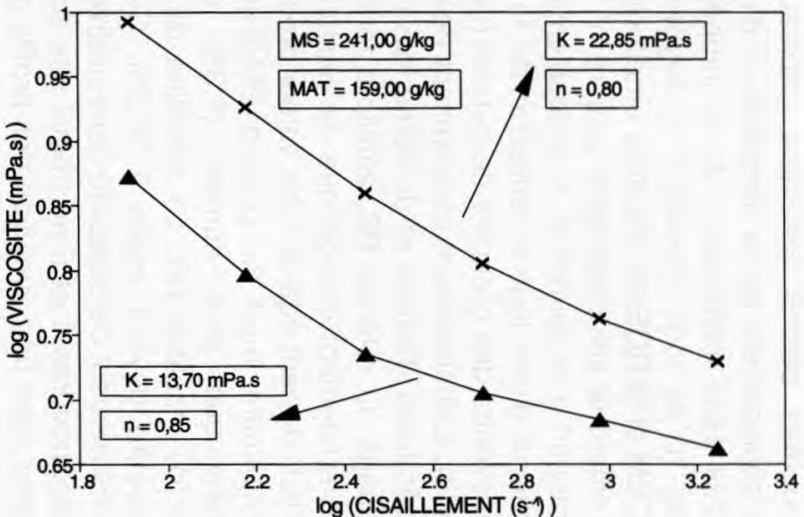

* R4 ESV (avant) $₫$ R4 ESV (après)

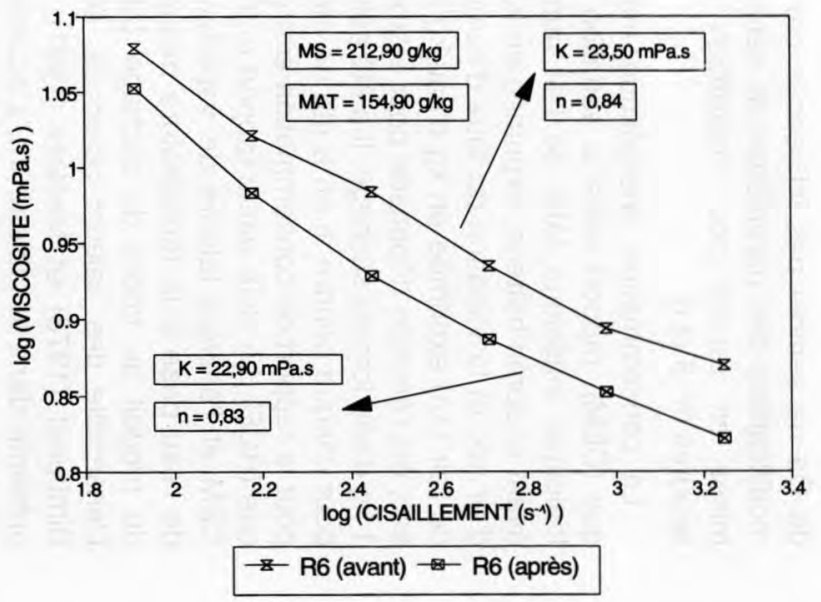


du Ca ne semble pas influencée par les modifications des paramètres de séchage mises en œuvre pour l'obtention des poudres $n^{\circ} 5$ et 6 .

La consommation énergétique massique (CEM), rapport entre la variation enthalpique massique $(\Delta \mathrm{H})$ de l'air humide avant et après batterie, exprimée en $\mathrm{kJ} / \mathrm{kg}$ d'air sec et la variation du taux d'humidité de l'air $(\Delta n)$ exprimée en $\mathrm{kg}$ d'eau/kg d'air sec, est l'énergie dépensée pour évaporer $1 \mathrm{~kg}$ d'eau lors du séchage. Il a été calculé pour chaque poudre. II en a été de même pour le rapport de consommation énergétique (RCE). Le RCE est le rapport entre la CEM et l'énergie latente de vaporisation de l'eau prise à la température moyenne du produit au cours du séchage $(\Delta \mathrm{Hv})$. L'ensemble des valeurs obtenues, selon Bimbenet (1978) et Masters (1991), est présenté dans le tableau VII. L'accroissement de la température d'entrée d'air, la température de l'air de sortie étant identique pour les poudres $n^{\circ} 2,3$ et 4 de rétentat, entraîne une diminution de la CEM et de la RCE, ce qui signifie une amélioration du rendement énergétique de l'installation de déshydratation. Les profils granulométriques des poudres (tableau VIII) n'en sont pas modifiés mais la solubilité des poudres décroît fortement (tableau IV). L'abaissement de la température d'entrée d'air sans modification de la température de l'air de sortie (poudre $n^{\circ} 5$ ) augmente la CEM et la RCE de $5 \%$ mais améliore légèrement la solubilité de la poudre $n^{\circ} 5$ par rapport à la poudre $n^{\circ} 4$, probablement en raison d'une légère réduction de la taille moyenne des granules de poudre (tableau VIII). L'abaissement concomitant des températures d'entrée et de sortie lors du séchage du rétentat $\mathrm{R} 6$, améliore fortement le rendement énergétique de l'installation, RCE devient égal à 1,30 , valeur proche de l'optimum mais la poudre obtenue est beaucoup plus humide $(8,3 \%$ contre $5,5 \%$; tableau IV). La solubilité de cette poudre devient égale à $97,2 \%$. Cet accroissement de solubilité comparativement aux poudres 4 et 5 ne trouve pas son origine dans une évolution des profils granulométriques (tableau VIII).

La comparaison des propriétés physiques des poudres de rétentats de microfiltration avec celles de la poudre de lait amène à la conclusion que la diminution de la matière sèche non protéique découlant du traitement du lait par microfiltration décroît la solubilité et la dispersibilité des poudres résultantes d'autant plus que le

Tableau VII. Consommation énergétique massique (CEM) et rapport de consommation énergétique (RCE) pour la poudre de lait écrémé de référence et les poudres de rétentat.

Specific heat consumption (CEM) and heat consumption ratio (RCE) for the reference skimmilk and retentate powders.

\begin{tabular}{|c|c|c|c|c|}
\hline Poudre & $\begin{array}{c}\Delta H \\
(\mathrm{~kJ} / \mathrm{kg} \text { d'air sec) }\end{array}$ & $\begin{array}{c}\Delta n \\
(k g \text { d'eau/kg d'air sec) }\end{array}$ & $\begin{array}{l}\text { CEM ( } \triangle H / \Delta n) \\
(k J / k g \text { d'eau) }\end{array}$ & $\begin{array}{c}R C E \\
(C E M / \Delta H V)\left(40^{\circ} \mathrm{C}\right)\end{array}$ \\
\hline
\end{tabular}

\begin{tabular}{lllll}
\hline 1 & 183 & 0,047 & 3894 & 1,62 \\
2 & 204 & 0,055 & 3709 & 1,55 \\
3 & 215 & 0,060 & 3583 & 1,49 \\
4 & 223 & 0,063 & 3540 & 1,47 \\
5 & 190 & 0,051 & 3725 & 1,55 \\
6 & 120 & 0,038 & 3158 & 1,31 \\
\hline
\end{tabular}


FCV est élevé. Pour des paramètres de déshydratation identiques, de plus faibles teneurs en molécules hygroscopiques (lactose, sels minéraux) doivent entraîner une cinétique accélérée d'élimination de l'eau à l'interface dans les gouttelettes formées lors de la pulvérisation (Webb et al, 1978). La conséquence de cette accélération du transfert de l'eau pourrait être une surconcentration des éléments de la matière sèche à l'interface gouttelette-air avec formation d'un film à la surface du granule de poudre résultant, peu perméable à la vapeur d'eau (Bimbenet et al, 1970). La solubilité et la dispersibilité à la reconstitution en seraient amoindries, l'eau de reconstitution ne pouvant pas pénétrer au sein du granule de poudre. La mouillabilité des poudres n'est quant à elle pas modifiée puisqu'elle ne dépend que du profil granulométrique (Pisecky, 1986).

Le ralentissement du transfert de l'eau par abaissement de la température d'entrée de l'air (poudre $n^{\circ} 5$ ) et de la température de l'air de sortie (poudre $n^{\circ} 6$ ) diminuerait ce phénomène de "coiffage" du granule de poudre et serait à l'origine des améliorations de solubilité et de dispersibi- lité constatées. Mais les valeurs atteintes restent inférieures à celles observées sur la poudre de lait de référence. Compte tenu des valeurs de CEM et de RCE atteintes pour les poudres $n^{\circ} 5$ et surtout $n^{\circ} 6$, il n'est pas envisageable de ralentir encore la vitesse de séchage de l'installation. L'amélioration des propriétés de solubilité et de dispersibilité des poudres de lait enrichies en caséine micellaire ne peut résulter que d'une modification de la composition biochimique des produits introduits en tour (accroissement de la teneur en molécules solubles hygroscopiques ou de la physico-chimie de l'interface goutteletteair : modification du $\mathrm{pH}$ ).

L'activité de l'eau $\left(\mathrm{a}_{\mathrm{w}}\right)$ a été déterminée sur les 6 poudres obtenues dès l'obtention et après $100 \mathrm{j}$ de conservation à température ambiante (tableau IV). Les $a_{w}$ des poudres 1 à 5 sont très voisines ; celle de la poudre $n^{\circ} 6$ est nettement supérieure du fait des conditions de séchage utilisées pour son obtention. L'accroissement de $\mathrm{l}^{\prime} \mathrm{a}_{\mathrm{w}}$ des 6 poudres au cours des $100 \mathrm{j}$ de conservation est du même ordre de grandeur. L'examen des isothermes d'adsorption (tableau IX) montre que la reprise

Tableau VIII. Profils granulométriques des différentes poudres de lait et de rétentat (\%). Granulometry profiles of the different milk and retentate powders (\%).

\begin{tabular}{lccccccccc}
\hline Poudre & $\begin{array}{c}<63 \\
(\mu \mathrm{m})\end{array}$ & $\begin{array}{c}63-80 \\
(\mu \mathrm{m})\end{array}$ & $\begin{array}{c}80-100 \\
(\mu \mathrm{m})\end{array}$ & $\begin{array}{c}100-160 \\
(\mu \mathrm{m})\end{array}$ & $\begin{array}{c}160-200 \\
(\mu \mathrm{m})\end{array}$ & $\begin{array}{c}200-250 \\
(\mu \mathrm{m})\end{array}$ & $\begin{array}{c}250-315 \\
(\mu \mathrm{m})\end{array}$ & $\begin{array}{c}315-400 \\
(\mu \mathrm{m})\end{array}$ & $\begin{array}{c}>400 \\
(\mu \mathrm{m})\end{array}$ \\
\hline 1 & 0 & 0,75 & 0,75 & 11,00 & 17,00 & 33,55 & 28,90 & 4,20 & 3,85 \\
2 & 0 & 0,25 & 0,15 & 11,45 & 22,55 & 43,40 & 20,30 & 1,05 & 0,85 \\
3 & 0 & 0 & 0,35 & 6,55 & 18,45 & 48,65 & 24,00 & 1,40 & 0,60 \\
4 & 0 & 0 & 0,60 & 2,75 & 12,50 & 45,75 & 34,40 & 3,65 & 0,35 \\
5 & 0 & 0 & 0,15 & 6,40 & 18,25 & 54,15 & 20,30 & 0,30 & 0,45 \\
6 & 0 & 0 & 0,70 & 9,30 & 21,70 & 47,55 & 19,35 & 0,80 & 0,60 \\
\hline
\end{tabular}


Tableau IX. Isotherme d'adsorption des poudres. Teneur en eau exprimée pour $100 \mathrm{~g}$ de matière sèche en fonction de l'humidité relative (\%).

Adsorption isotherm of the powders. Water content expressed for $100 \mathrm{~g}$ of total solids in function of relative moisture (\%).

\begin{tabular}{lllllllll}
\hline \multirow{2}{*}{ Poudre } & \multicolumn{7}{c}{ Humidité relative (\%) } \\
\cline { 2 - 8 } & 8 & 11 & 23 & 33 & 53 & 75 & 90 & 98 \\
\hline 1 & 0 & 0,91 & 2,00 & 3,82 & 10,38 & 19,93 & 31,96 & 95,53 \\
2 & 0 & 0,92 & 1,77 & 3,62 & 9,17 & 18,78 & 32,26 & 68,77 \\
3 & 0 & 1,21 & 2,11 & 3,76 & 8,69 & 18,05 & 31,82 & 68,00 \\
4 & 0 & 0,96 & 3,45 & 4,55 & 8,25 & 16,78 & 30,38 & 64,68 \\
5 & 0 & 1,22 & 2,59 & 4,65 & 8,07 & 15,79 & 30,66 & 64,30 \\
6 & 0 & 1,01 & 2,08 & 4,70 & 6,90 & 15,79 & 30,15 & 60,44 \\
\hline
\end{tabular}

d'eau est voisine pour les poudres $n^{\circ} 1,2$ et 3 jusqu'à $33 \%$ d'humidité relative (HR). II en est de même pour les poudres $n^{\circ} 4,5$ et 6 , mais avec une valeur légèrement supérieure. Au-delà de $33 \%$ d'HR, la reprise d'eau des poudres de lait enrichies en caséine micellaire diverge fortement de celle observée pour la poudre de lait de référence. La différence de comportement des poudres 1 à 3 comparativement aux poudres 4 à 6 dans la partie inférieure des isothermes ( $\mathrm{HR}<33 \%$ ) est probablement due aux différences de teneurs en protéines des poudres. En effet, selon Cheftel et Cheftel (1976) l'hygroscopicité des protéines est supérieure à celle des glucides aux faibles HR. Au-delà de $33 \%$ d'HR, la réduction notable de reprise d'eau par les poudres de rétentat par rapport à la poudre de référence découle probablement aussi de la différence de teneur en glucides fortement hygroscopiques aux HR élevées (Cheftel et Cheftel, 1976). Dans les poudres de rétentat, la mise en contact avec l'air humide, comme lors de la reconstitution, se traduit pas une hydratation de surface avec formation d'une couche protéique visqueuse peu perméable, limitant l'hydratation interne.

L'évolution dans le temps des caractéristiques physiques des poudres (tableau IV) montre également une très nette différence de comportement entre la poudre de lait de référence et les poudres enrichies en caséine micellaire. Les propriétés de solubilité, de dispersibilité et de mouillabilité de la poudre de lait ne se dégradent pas en dépit d'une légère reprise d'humidité. Ces résultats sont conformes à ceux de De Vilder (1982). Par contre, les poudres de rétentat voient leurs solubilités diminuer de $0,2 \%$ (poudre $n^{\circ} 2$ ) à $9,8 \%$ (poudre $n^{\circ} 5$ ) et leurs dispersibilités décroître de $1,5 \%$ (poudre $n^{\circ} 2$ ) à $14,0 \%$ (poudre $n^{\circ} 3$ ). Cette dégradation des caractéristiques physiques est moins élevée pour la poudre $n^{\circ} 6$ mais l'indice de dispersibilité reste très éloigné de celui observé pour la poudre 
$n^{\circ} 1$. Cette évolution dans le temps des caractéristiques physiques des poudres de rétentat est certainement provoquée par les mêmes mécanismes d'interface évoqués au cours des observations réalisées lors de la détermination des isothermes d'adsorption. Le comportement différent de la poudre $n^{\circ} 6$ découle probablement d'une teneur initiale en eau très supérieure à celle des poudres similaires.

L'aptitude à la coagulation par la présure des laits reconstitués à partir des poudres a été appréciée par la mesure des temps de coagulation $(R)$, de cinétique de raffermissement $\left(K_{20}\right)$ et de fermeté $(a R)$ (tableau X). L'épuration bactérienne par microfiltration n'influence pas l'aptitude à la coagulation par la présure du lait. L'enrichissement du lait en caséine micellaire suivi d'une dilution aqueuse (les rétentats ont été ramenés à $25 \mathrm{~g} / \mathrm{l}$ de caséine), ce qui conduit à une dilution de la phase soluble et à une diminution de la proportion de protéines solubles, se traduit par une diminution significative du temps de prise ( $-36 \%$ pour R2 ; $-42 \%$ pour R4 ; $-50 \%$ pour R6), une accélération du $\mathrm{K}_{20}$ de $5 \%$ à $30 \%$ et une diminution de la fermeté de 13 à $18 \%$. Ces observations sont en parfait accord avec celles de Pierre et al (1992) et découlent de l'élimination par microfiltration des éléments de la phase soluble du lait exerçant un effet défavorable sur la coagulation enzymatique. Dans les conditions de mesure, la concentration par évaporation et la déshydratation par atomisation n'apparaissent pas avoir d'effets significatifs sur les 3 paramètres mesurés : $R, K_{20}$ et aR sauf pour ce qui est du rétentat $\mathrm{R} 2$ pour lequel l'évaporation sous vide induit une dégradation des aptitudes à la coagulation par la présure, mais

Tableau X. Aptitude à la coagulation des différents produits obtenus pour la fabrication des poudres de rétentat.

Coagulation properties of the different products obtained for the manufacture of the retentate powders.

\begin{tabular}{lrrr}
\hline Produit ${ }^{1}$ & Temps de prise $(R)(\mathrm{min})$ & $K_{20}(\mathrm{~min})$ & Fermeté $(\mathrm{aR})(\mathrm{mm})$ \\
\hline Lait écrémé & 18,87 & 10,31 & \\
Lait écrémé épuré & 18,75 & 10,25 & 28,30 \\
& & & 28,10 \\
Rétentat R2 & 11,90 & 9,75 & 23,80 \\
Rétentat R4 & 10,87 & 9,00 & 23,00 \\
Rétentat R6 & 9,33 & 7,16 & 24,50 \\
& 13,00 & 11,40 & 20,12 \\
Rétentat R2 ESV & 10,47 & 8,54 & 22,50 \\
Rétentat R4 ESV & 13,13 & 10,70 & 24,90 \\
& 10,58 & 8,97 & 23,30 \\
Poudre No2 & 9,65 & 7,15 & 24,10 \\
Poudre N03 & & & \\
Poudre N*4 & & & \\
\hline
\end{tabular}

\footnotetext{
1 Tous ces produits ont été reconstitués et standardisés en caséines à $25 \mathrm{~g} \cdot \mathrm{l}^{-1}$ dans de l'eau distillée.

${ }^{1}$ Products reconstituted and standardized in casein to $25 \mathrm{~g} \cdot \mathrm{r}^{-1}$ in distilled water.
} 
cette dégradation ne se retrouve que partiellement dans le lait reconstitué à partir della poudre $n^{\circ} 2$. Ce comportement du rétentat R2 ESV est probablement à relier avec l'évolution du rapport $\mathrm{Ca} . \mathrm{s} / \mathrm{Ca}_{\mathrm{t}}$ au cours de l'évaporation (tableau VI).

\section{REMERCIEMENTS}

Nous remercions MN Madec et R Pannetier d'avoir effectué les analyses microbiologiques et chimiques, ainsi que Y Le Graet et A Pierre pour les précieux conseils apportés dans la rédaction de cette publication.

\section{RÉFÉRENCES}

A.cton GH (1977) The determination of the lactose in cheese. Aust J Dairy Technol 32, 111-112

AFNOR (1984) Lait de conserve. Microbiologie. Norme NF V 04-015

Alais C (1984) Sciences du lait. Principes des techniques laitières. SEPAIC, Paris, $4^{e}$ edn, 734-735

Amariglio S (1986) Contrôle de la qualité des produits laitiers. Analyses physiques et chimiques. AFNOR, ITSV, Paris, $3^{e}$ ed, 439445

AOAC Standards (1945), 559

Ashaffenburg R, Drewry J (1959) New procedure for the routine determination of the various non casein proteins of milk. Int Dairy Congr 3(5) 1631-1637

Bimbenet JJ (1978) Le séchage dans les industries agricoles et alimentaires. SEPAIC, Paris, $31 \mathrm{p}$

Bimbenet JJ, Brusset H, Loncin M (1970) Effet de la présence de corps solubles sur la déshydratation des produits biologiques. Ind Alim Agric 87, 385-391

Bloore C, Boag I (1982) The effect of processing variables on spray dried milk powder. NZJ Dairy Sci Technol 17, 103-120

Borios M, Lancelot J, Davenas P, llari JL (1984) Vérification industrielle de l'influence de certains paramètres technologiques de fabrica- tion sur les propriétés physiques de la poudre de lait écrémé. Ind Alim Agric 101, 1211-1219

Brulé G, Maubois JL, Fauquant J (1974) Étude de la teneur en éléments minéraux des produits obtenus lors de l'ultrafiltration du lait sur membrane. Lait 54, 600-615

Cheftel JC, Cheftel H (1976) Introduction à la biochimie et à la technologie des aliments (1), Technique et Documentation, Paris, 6-23

Culioli J, Bon JP, Maubois JL (1974) Étude de la viscosité des rétentats et des préfromages obtenus après traitement du lait par ultrafiltration sur membrane. Lait 54, 481-500

De Vilder J (1982) Altérations en cours de conservation des caractéristiques du lait en poudre. Rev Agric 35, 2051-2064

De Vilder J (1986) La fabrication de poudre de lait écrémé instantanée. Rev Agric 39, 865877

Fauquant J, Maubois JL, Pierre A (1988) Microfiltration du lait sur membrane minérale. Tech Lait 1028, 21-23

Ferron-Baumy C, Maubois JL, Garric G, Quiblier JP (1991) Coagulation présure du lait et des rétentats d'ultrafiltration. Effets de divers traitements thermiques. Lait 71, 423-434

FIL-IDF (1988) Détermination de l'indice d'insolubilité. Norme $n^{\circ} 129 \mathrm{~A}$

Ilari JL, Loisel C (1991) La maîtrise de la fonctionnalité des poudres. Process 1063, 39-43

Le Graet Y, Brulé G (1982) Effet de la concentration par évaporation et du séchage sur les équilibres minéraux dans le lait et les rétentats. Lait 62, 113-125

Mc Mahon DJ, Brown RJ (1982) Evaluation of Formagraph for comparing rennet solutions. J Dairy Sci 65, 1639-1642

Masters K (1991) Spray drying, 5th ed. Longman Scientific \& Technical and John Wiley \& Sons Inc, Essex

Maubois JL (1991) New applications of membrane technology in the dairy industry. Aust $J$ Dairy Technol 46, 91-95

Maubois JL, Fauconneau G (1977) Les choix possibles dans le domaine de la technologie laitière pour résoudre le problème des excédents laitiers. Journées ITEB, 141-150

Morand A (1975) Le séchage par pulvérisation. Journées CPCIA, 4-14 
Murthy GK, Rhea U (1967) Determination of major cations in milk by atomic absorption spectrophotometry. J Dairy Sci 50, 313-317

Niro Atomizer (1978) Méthodes d'Analyses des Produits Laitiers Déshydratés. $4^{\mathrm{e}}$ edn

Pierre A, Fauquant J, Le Graet Y, Piot M, Maubois JL (1992) Préparation de phosphocaséinate natif par microfiltration sur membrane. Lait 72, 461-474

Pisecky J (1986) Standards, specifications and test methods for dry milk products. In: Concentration and drying of foods (Mac Carthy D, ed). Elsevier, London, 203-220

Rowland SJ (1938) The determination of nitrogen distribution in milk. J Dairy Res 9, 4246
Trouvé E, Maubois JL, Piot M, Madec MN, Fauquant J, Rouault A, Tabard J, Brinkman G (1991) Rétention de différentes espèces microbiennes lors de l'épuration du lait par microfiltration en flux tangentiel. Lait 71, 1-13

Tuohy JJ (1989) Some physical properties of milk powders. Ir J Food Sci Technol 13, 141-15ู2

Vincens D, Tabard J (1988) L'élimination des germes microbiens sur membrane de microfiltration. Tech Lait Market 1033, 62-64

Weeb BH, Johnson AH, Alfort JA (1978) Fundamentals of dairy chemistry. 5nd edn, Avi Publishing Co, Wesport

Zannoni M, Anibaldi S (1981) Standardization of the renneting ability of milk by Formagraph. Sci Tec Latt-Casearia 32, 79-94 